\title{
Active Insulin Infusion Control of the Blood Glucose Derivative
}

\author{
J. Geoffrey Chase, Z-H Lam, J-Y Lee and K-S Hwang \\ Dept of Mechanical Engineering \\ University of Canterbury \\ Private Bag 4800 \\ Christchurch, New Zealand \\ Email: g.chase@mech.canterbury.ac.nz
}

\begin{abstract}
Close control of blood glucose levels significantly reduces vascular complications in Type I diabetes. A control method focusing on the rate of change of blood glucose level is developed to utilize emerging technologies in blood glucose biosensors. The controller developed provides tighter, more optimal control of blood glucose levels, while robustly handling variation in patient response and sampling rate. Particular emphasis is placed on the controller simplicity and robustness necessary for medical devices and implants. A PD controller with a heavily weighted derivative term is found to outperform the more proportional-weighted controllers in oral glucose tolerance testing. Simulation results show reductions of over $50 \%$ in the magnitude and duration of blood glucose excursions from basal levels that are slightly better than normal non-diabetic response as modelled. Comparison with normal response indicates that the physiological control system has some measure of both proportional and derivative control as the basis of glucose regulation.
\end{abstract}

\section{Introduction}

Improperly managed, diabetes can lead to complications such as nerve damage, brain damage, amputation and eventually death. Diabetes related complications are a worldwide epidemic with high medical, economic and social costs [1-3]. Tight control of blood glucose levels has also been shown to reduce the mortality of diabetic, and non-diabetic, intensive care unit patients by up to $50 \%$ [4].

Diabetic individuals monitor food intake and daily activity to maintain blood sugar levels at an adequate level. For ease of management, subjects are encouraged to stick to strict routines and diets to minimize manual monitoring and injections, reducing intervention and difficulty. However, this regime can lead to severe limitation of the subjects' lifestyle, an "institutional" psychology, and the difficulty of consistently maintaining a strict daily regimen over several years.
Though devices that can measure glucose level and administer insulin exist, they do just that - measure and inject - with no automated interface between the two. Current treatment for Type I diabetes involves monitoring the plasma glucose level and injecting insulin as needed. Normally, patients follow a strict regimen to prevent complications, however the effectiveness of this regimen is a function of the patient's intuition and experience. A typical day for a diabetic individual might involve injecting long-acting insulin approximately three times and injecting rapidacting insulin before meals, to reduce the post-prandial blood glucose spike. The patient is required to make intuitive decisions when they deviate from normal diet or exercise patterns, and modify their regimen to suit the irregularity. As a result, error is introduced and control is often not optimal.

Most commonly available glucose sensing devices operate by measuring the blood glucose content of a small finger-prick blood sample, an irritating method upon frequent use. As a result, some diabetic individuals measure blood sugar as infrequently as once per day, or less. However, recent advances have resulted in semi-invasive systems such as the GlucoWatch Biographer from Cygnus [5]. This device offers sampling rates up to one reading every 20 minutes, and can measure and store data continuously for up to 12 hours before new sensor pads are required.

Current diabetes management typically seeks to limit blood glucose levels to less than 5.8-6.0 mmol/L, however tighter control to the basal level of 4.5-5.0 $\mathrm{mmol} / \mathrm{L}$ would significantly limit the damage that results from long-term exposure to elevated blood glucose levels. The acceptance of higher blood glucose levels is considered acceptable, as blood glucose management without advanced sensors does not deliver the data or the capability to constantly modify insulin infusion rates to achieve tighter control. However, regular, automated blood glucose measurement provides the consistent volume of data necessary for such tight control, in contrast to the sometimes infrequent and inconsistent self-measurement reported by clinicians.

Automation can also have a significant impact on the treatment of juveniles with Type I diabetes as children have less ability to fully manage the disease and, as a 
result, the stress and difficulty of management can fall on parents and others. In all cases, an automated approach can provide clinical staff with a far greater abundance, and consistency of data, enabling more effective treatment and feedback.

Whether it is in the area of understanding, modeling or managing diabetes [6,7] years of research in this area has led to no shortage of theoretical solutions [8-17]. However, due to either the complexity of the proposed implementation, current technological limitations, models that are not physiologically verified, lack of required data, or the cost/complexity of implementing the results, these solutions are not yet fully practicable.

Several researchers have examined the analysis and automation of insulin as reviewed by Lehman et al. [18]. Many of the systems presented use control as a means of providing clinical advice or testing the efficacy of a new protocol [19-24]. A more complex, higher performance real-time control example uses model predictive control on a 19th order system identified model of the glucose-regulatory system resulting in a $40 \%$ peak reduction and $23 \%$ reduction in settling time to basal level [25]. Optimal control using grid search theory, robust H-infinity control, and variable structure controllers have also been studied, each using different models [11, 24-28]. In each case, the focus has been on controlling absolute blood glucose excursion rather than slopes. The models used typically require either patient specific parameters that are not readily available and/or knowledge of glucose or exercise inputs that would not be known a priori for an ambulatory individual. Finally, none study the impact of more frequent measurement enabled by recent advances in sensing technology or the potential for improved results.

\section{Glucose Regulatory System Model}

Comprehensive models, though very accurate for regimen evaluation, are generally unsuited for real-time control, requiring several time points of input to generate the insulin infusion profile. Additionally, they are not generic requiring patient-specific data and known glucose inputs. The aim of this research is to develop control schemes based on models that capture the essential system dynamics, do not require unavailable data, and are applicable to a wider variety of subjects. Simple models capture these essential dynamic behaviours, providing a more suitable foundation for real-time control design and analysis.

A well known, and more importantly, physiologically verified model originated from the work of Bergmann et al. [15]. It utilises the concept of a remote compartment for the storage of insulin to account for the time delay between injection of insulin and its utilization to reduce blood glucose levels. Equations (1)-(3) show the equations used to define the system.

$$
\begin{aligned}
& \dot{G}=-p_{1} G-X\left(G+G_{B}\right)+P(t) \\
& \dot{X}=-p_{2} X+p_{3} I \\
& \dot{I}=-n\left(I+I_{B}\right)+u(t) / V_{I}
\end{aligned}
$$

Where $G$ is the plasma glucose concentration over basal $\left(\mathrm{mMol} \mathrm{L} \mathrm{L}^{-1}\right), G_{B}$ is the basal plasma glucose ( $\mathrm{mMol}$ $\left.L^{-1}\right), X$ is the generalized insulin variable for the remote compartment $\left(\mathrm{min}^{-1}\right), I$ is the insulin concentration above basal $\left(m U L^{-1}\right), I_{B}$ is the basal insulin level $(m U$ $\left.L^{-1}\right), P(t)$ is the external glucose input $\left(\mathrm{mMol} \mathrm{L}^{-1} \mathrm{~min}^{-1}\right)$, $u(t)$ is the external insulin input $\left(m U L^{-1} \mathrm{~min}^{-1}\right), p_{3}$ is a patient parameter $\left(m U^{-1} L \mathrm{~min}^{-2}\right), V_{I}$ is the insulin distribution volume $(L)$, and $n, p_{1}, p_{2}$ are patient specific parameters $\left(\mathrm{min}^{-1}\right)$. The parameters, $p_{1}, p_{2}$ and $p_{3}$, may be changed to represent different conditions and for Type I diabetic individuals: $p_{1}=0, p_{2}=0.025$, $p_{3}=0.000013[10,13,15]$.

The model is simple, yet accurately represents the essential dynamics of the human glucose regulatory system. The three equations represent insulin production and infusion, insulin storage in a remote compartment, and glucose input and insulin utilization in a second compartment. The values of $n, V_{I}, G_{B}, I_{B}$ employed are defined, for an average-weighted male as: $V_{I}=12 \mathrm{~L}, n=5 / 54 \mathrm{~min}^{-1}, G_{B}=4.5 \mathrm{mmol} \mathrm{L}^{-1}, I_{B}=$ $15 \mathrm{mU} \mathrm{L}^{-1}[10]$.

The controller uses a simple feedback loop that employs the blood glucose level above basal, $G$, and its derivative, $\dot{G}$, as sensor inputs, and the exogenous insulin infusion rate, $u(t)$, as the control output. As a result, the controller measures the output from Equation (1) while directly influencing Equation (3) via the control action. In between are the time delay and dynamics of the remote compartment defined in Equation (2).

\section{Controller Definition}

There are many complex influences between glucose and insulin concentration for any person, normal or diabetic. However, the steady state glucose concentration in the body is finally determined by how much insulin is present. In order to lower glucose concentration in the blood, insulin needs to be released, injected or infused. Hence, the controller defines the insulin infusion rate, $u(t)$, based on the measured inputs $G$ and/or $\dot{G}$. The goal is to minimise excess glucose, $G$, and its rate of change, $\dot{G}$, and ensure that excursions from the basal value, $G_{b}$, are minimised in magnitude and duration with no hypoglycemic overshoot below the basal level. Therefore, blood sugar levels must be maintained in a tight range around the 
basal level, which matches the concept that the ideal blood glucose curve should be relatively, if not completely, flat [3].

\subsection{Relative Proportional Control (RPC)}

One of the better-known, more effective diabetes control systems is a relative proportional control law (RPC) [10]. This basic, widely used form of proportional control law is based on the idea of strictly limiting the absolute blood sugar level by applying resisting "forces" (insulin) in weighted proportion to the magnitude of the excursion from the desired (basal) level. The control inputs are defined:

$$
u(t)=u_{0}\left(1+\frac{G}{G_{b}}\right), \quad u_{0}=n V I_{b}
$$

This controller is based on a relative proportional control $\left(G / G_{b}\right)$ with a constant term. Note that when $G$ $=G_{b}$ the blood sugar is at the desired level and the insulin infusion rate is $u_{0}$, the basal infusion rate necessary to maintain blood glucose at a constant level.

\subsection{Heavily Weighted Derivative Controller}

This controller uses a PD control law where the derivative gain is large and the proportional gain is much smaller.

$$
u(t)=u_{0}\left(1+k_{p} G+k_{d} \dot{G}\right)
$$

Where Equation (5) incorporates proportional and derivative control with independent gains to weight the different terms and the basal infusion $u_{0}$.

Heavy emphasis implies that the derivative gain, $k_{d}$, is significantly larger than the proportional gain, $k_{p}$. As a result, this controller focuses almost exclusively on controlling the slope of the blood glucose curve rather than its absolute magnitude. This approach is far different than normally taken and made possible by the emerging capability to measure blood sugar far more regularly via semi- or non- invasive methods. The gains will of course vary with sensor sampling rate.

There are several benefits provided by derivative emphasized control for this non-linear, delayed system. Most importantly, a large increase in glucose is observed before a large proportional term, enabling a faster response time for the controller. This approach implies that the sooner insulin is infused, or injected, the earlier it can be utilized to reduce blood glucose analogous to the thinking behind current diabetes injection regimes. In addition, flattening the slope of the glucose response provides an effective, implicit means of minimizing the (eventual) magnitude of the entire glucose response before it rises to high. Derivative terms can be susceptible to noise, but in this case with appropriate filtering and technology it should not be an issue.

\section{Numerical Tests and Verification}

This section presents the numerical verification of the control systems presented by simulating oral glucose tolerance testing. Comparisons are also made to normal human response.

\subsection{Oral Glucose Tolerance Test}

The oral glucose tolerance test (OGTT) is a large step input test of the glucose regulatory system often performed to diagnose diabetes. A fasting subject consumes 400-800 calories of glucose and the response is observed. This beta-cell function test represents a significant challenge to the pancreas and measures the insulin response capability. The OGTT glucose input may be modelled by the lognormal distribution defined in Equation (6).

$$
P(t)=P_{m} e^{-a(\ln (b t)-c)^{2}}
$$

Where $P_{m}$ is the peak value and $a, b$ and $c$ are constants, which determine the exact shape. This function is employed because it is smooth, continuously differentiable, has zero initial conditions, is easily implemented, and physiologically accurate. It is easily modified to represent different absorption rates of exogenous glucose.

Figure 1 shows the glucose response for an OGTT using the PD and RPC controllers with sensor measurements every 1 and 20 minutes. There are also lines for the accumulated glucose input and normal, non-diabetic response.

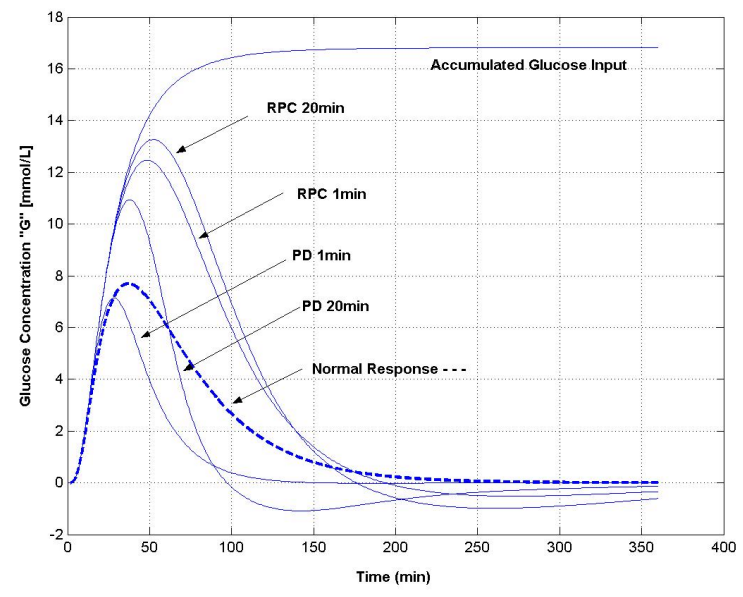

Figure 1: Glucose response for an OGTT. 
Figure 2 shows the insulin infusion profile for the RPC and PD controller responses shown in Figure 1. The step function shape is due to the 20-minute sampling interval employed and the resulting 20-minute interval between changes in infusion rate. Note that as the sampling interval shrinks the insulin profiles for the PD controllers get smoother and sharper, with a shape more like that of an insulin injection profile.

In judging controller performance the critical factors are the magnitude of the excursion for a given input and the time required to return to basal blood sugar levels. The PD controller limits the excursion and returns to the basal level much faster, in each case, due to the higher infusion rates generated by large initial slopes of the glucose curve. Figure 2 also shows that as the sampling rate increases, the insulin infusion profile for the PD controller tends to mimic the sharper spike of an injection profile. These results match current methods of managing diabetes that have evolved over several decades, where insulin is injected before meals while long acting insulin is used to mimic the basal infusion rate, $\mathrm{u}_{0}$, when there is no external glucose input.

Sensor bandwidth, or sampling period, is varied since the current GlucoWatch measures blood sugar levels every 20 minutes. The one-minute bandwidth graph shows what can be achieved when technology enables measurements at this rate. The 1-minute plots are more useful when considering the feasibility of simple controllers for implanted units with direct, regular access to blood sugar level measurements. Smaller sampling periods have less impact as the sampling period becomes small relative to system time constants that are larger than 1 minute.

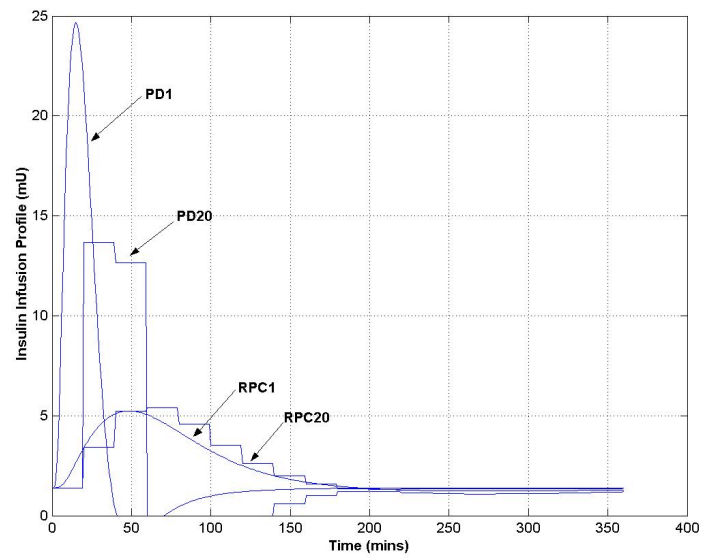

Figure 2: Insulin infusion rate for an OGTT

The PD controller shows a greater loss of performance as the sampling interval rises because the derivative values lose their usefulness as the time between samples grows. This result, as evidenced in Figure 1, illustrates the impact of new sensor technology and the improvements it can enable in glucose response performance. Specifically, current practice with infrequently available data does not allow effective use of the derivative term. New sensor technology enables more optimal control of blood glucose level as steep slopes in the glucose response can be employed to accurately sense forthcoming glucose spikes and provide effective insulin infusion to counter them.

The comparison to normal glucose response is also important. Figure 1 shows that the PD controller, due to its rapid insulin input, outperforms normal response in terms of both magnitude and duration of the glucose excursion from basal level. The RPC controllers both have response similar to the normal curve, but with higher magnitude, indicating that the human glucoregulatory system is likely a primarily proportional based control system. The 20-minute sampling interval PD controller has higher magnitude, as discussed previously, but much shorter duration. However, this difference is offset by a small amount of hypoglycemic overshoot for the PD controller that results from the longer sampling interval in the feedback loop.

Finally, a GlucoWatch sensor measurement lags approximately one sample period due to the method by which it obtains the value. Simulation with this additional lag was simulated with a 20-minute sampling period for the PD controller to determine its impact on control efficacy. As expected, control response lags further allowing greater glucose excursions in both magnitude and duration when compared to the results shown in Figure 1. However, this lag impacts the results of proportional and derivative weighted controllers alike. Similar results are seen when accounting for 1-3 sample period failures in the sensor.

\subsection{Comparison of Control Methods}

It is seen that the heavy derivative PD controller outperforms the RPC. The RPC definition in Equation (4) has a gain of 1.0 on the relative proportional term. However, as this gain is increased for better peak performance hypoglycemic overshoot grows because even as the glucose levels are decreasing toward basal a proportional controller is still infusing insulin. The result is hypoglycemia due to over-insulinising a system with significant delay. Hence, simply increasing proportional gain is not a practicable solution, in general unless explicitly and exactly accounting for the time delays of the system.

The PD controller, which is able to command significant insulin infusion earlier than a proportional dominated controller, provides better peak response control and limits hypoglycemic overshoot. Due to the large derivative gain the PD controller cuts the insulin infusion to zero when $\dot{G}$ is significantly negative enabling a more gentle drift back to the basal level when the glucose level starts dropping. This approach 
enables better control and, with greater sensor bandwidth, drives the control input to match current, effective diabetes management with infusion profiles that are essentially pre-meal injections with a basal infusion rate at other times.

In terms of the insulin infusion profiles shown in Figure 2 the primary difference is the lower, flatter profile for the RPC controller. While the PD controller mimics injections the RPC controller mimics a more constant infusion. Note that the area under these curves is approximately the same for each controller with the same sampling interval. So the same amount of insulin is employed, however the different controllers employ this amount differently to obtain different results.

Overall, both control methods presented are found to be both simple and effective. This control approach is less affected by patient specific parameters and as a result parameter variation is better tolerated for both controllers. Different types of insulin however cause difficulty where the control gains are tuned or a time constant that models insulin that acts in approximately 12 minutes, with faster or slower acting insulin employed in simulation.

\section{Conclusions}

The emergence of glucose sensors capable of providing blood glucose readings at a very high rate is investigated for their ability to automate insulin infusion for diabetic individuals, by opening the possibility of controlling the slope of the glucose response curve, rather than focusing strictly on absolute blood glucose level. A simple PD controller where the derivative term heavily outweighs the proportional term is shown to be highly effective. A proportional-based controller is also simulated to highlight the effectiveness of controlling the slope of the glucose curve rather than the absolute blood glucose level. The impact of different sampling periods, sensor lag, and the relative impact of proportional and derivative control is delineated.

The primary result is that a simple PD controller, heavily emphasising the derivative term, can effectively and robustly manage a variety of glucose input profiles slightly better than even a normal person. More specifically, the PD controller is shown to control glucose excursions up to $50 \%$ better than proportionalbased control schemes and slightly better than the normal human system as represented by the model employed. The comparisons with the normal physiological response indicates that an approximation of the normal physiological glucose regulation system is primarily proportional control with some measure of derivative control to eliminate or minimize any hypoglycemic overshoot.
Finally, as the sampling period drops, the insulin infusion profiles for this type of PD controller mimic current diabetes management utilizing pre-meal injections and low infusions at other times, verifying the basic approach. As a result, far tighter control of blood glucose excursions from basal levels can be used to reduce the impact of long-term exposure to (slightly) elevated blood glucose levels and increase the consistency of treatment. Ongoing research is focused on developing the protocol for testing this algorithm under controlled circumstances using ICU and ambulatory diabetic individuals.

\section{References}

[1] Diabetes New Zealand Inc. (2001). "Type II Diabetes: Managing for Better Health Outcomes" PriceWaterHouseCoopers Report. S. Thomson, Prof. D. Beaven, M. Jamieson, S. Snively, A. Howl, A.Christophersen

[2] American Diabetes Association. "Economic consequences of Diabetes Mellitus in the US in 1997". Diabetes Care 1998 21:296-309

[3] Alberti KGMM \& Zimmett P Z. Definition, "Diagnosis and classification of diabetes Mellitus - provisional report of a WHO consultation group". Diabetes Med. 1998 $15: 539-53$

[4] Van den Berghe, G, Wouters, P. Weekers, F., Verwaest, C., Bruyninckx, F., Schietz, M., Vlasselaers, D., Ferdinance, P., Lauwers, P. and Bouillon, R. (2001). "Intensive insulin therapy in critically ill patients," New England J. of Med., vol. 345(19), pp. 1359-1367.

[5] Garg, SK, Potts, RO, Ackerman, NR; Fermi, S, Tamada, JA and Chase, PH, (1999). "Correlation of finger-stick blood glucose measurements with GlucoWatch ${ }^{\circledR}$ biographer glucose results in young subjects with Type 1 diabetes," Diabetes Care 22:1708.

[6] Worthington, D. (1990). "Use of models in the self-management of insulin-dependent diabetes mellitus," Computer Methods and Programs in Biomedicine, vol. 32(3-4), pp. 233-239.

[7] Parker, R., Doyle, F., Harting, J., and Peppas, N. (1996). "Model predictive control for infusion pump insulin delivery," IEEE Conf. Of Engineering in Medicine and Biology, Nov 3, pp. 1822-1823.

[8] Doyle, F., Dorski, C., Harting, J., and Peppas, N. (1995). "Control and modeling of drug delivery devices for the treatment of diabetes," Proc. of 
the American Control Conference, June 21-23, pp. 776-780,

[9] Ollerton, R. (1989). "Application of optimal control theory to diabetes mellitus," Intl. Journal of Control, vol. 50(6), pp. 2503-2522.

[10] Kienitz, K. and Yoneyama, T. (1993). "Robust controller for insulin pumps based on $\mathrm{H}$-infinity theory," IEEE Trans. On Biomedical Engineering, vol. 40(11), pp. 1133-1137.

[11] Fisher, Michael E., (1991). “A Semiclosed-Loop Algorithm for the Control of Blood Glucose Levels in Diabetics", IEEE Transactions on Biomedical Engineering, vol 38, No. 1 pp 57-61

[12] Trajonoski, Z. and Wach, P. (1995). "Evaluation of subcutaneous route for the closed-loop control of insulin delivery: Numerical and experimental studies," IEEE Conf. on Engineering in Medicine and Biology, Sep 2023, pp. 1357-1358.

[13] Cobelli, C., Nucci, G., and Del Prato, S. (1999). "Physiological simulation model of the glucoseinsulin system," IEEE Conf. on Engineering in Medicine and Biology, Oct 16, pp. 999.

[14] Furler, Stuart M., Kraegen, Edward W., Smallwood, Robert H., and Chisholm, Donald J. (1985). "Blood Glucose Control by Intermittent Loop Closure in the Basal Mode: computer Simulation Studies with a Diabetic Model", Diabetes Care, Vol. 8, No. 6 pp 553 - 561.

[15] Bergman, Richard N., Finegood, D. T., and Ader, M., (1985) "Assessment of Insulin Sensitivity in Vivo", Endocrine Reviews, vol 6, no 1 , pp 45- 86 .

[16] Kraegen, E. W. and Chisolm, D. J., (1984), "Insulin responses to carting profiles of subcutaneous insulin infusion: Kinematics modelling studies", Diaetologia, vol. 26, pp 208-213.

[17] Cobelli, C., Federspil, G., Pacibi, G., Salvan, A., and Scandellari, S. (1982) "An Integrated Mathematics Model of the Dynamics of Blood Glucose and Its Hormonal Control" Mathematical Biosciences, vol 58 pp 27 - 60.

[18] Lehman, E. and Deutsch, T. (1996). "Computer assisted diabetes care: a 6-year retrospective," Computer Methods and Programs in Biomedicine, vol 50, pp. 209-230.

[19] Schrezenmeir, J. (1991). "Computer aids in dietetics," Diabetes, Nutrition and Metabolism, Supplement 1, pp. 175-191.

[20] Skyler, J., Skyler, D., Seigler, D. and Sullivan, M. (1981). "Algorithms for adjustment of insulin dosage," Diabetes Care, vol 4, pp. 311318.
[21] Lehman, E. and Deutsch, T. (1993). "AIDA: a mk. II automated insulin dosage advisor," Journal of Biomedical Engineering, vol 15, pp. 201-211.

[22] Boroujerdi, M., Williams, C., Carson, E., Piwernetz, K., Hepp, K., and Sonksen, P. (1987). "A simulation approach for planning insulin regimes," Intl. Symposium on Advanced Models for Therapy of Insulin Dependent Diabetes, pp. 41-46.

[23] Fletcher, L. and Soden, P. (1991). "Diet construction using linear programming," Diabetes, Nutrition and Metabolism, Supplement 1, pp. 169-174.

[24] Berger, M. and Rotbard D. (1991). "A pharmacodynamic approach to optimising insulin therapy," Computer Methods and Programs in Biomedicine, vol 34, pp. 241-253.

[25] Parker, R., Doyle, F., Harting, J. and Peppas, N. (1996). "Model predictive control for infusion pump insulin delivery," IEEE Conf on Eng in Med and Biology, pp. 1822-1823.

[26] Ollerton, R. (1989). "Application of optimal control theory to diabetes mellitus," International Journal of Control, vol 50(6), pp. 2503-2522.

[27] Naylor, J., Hodel, A., Morton, B., and Schumacher, D. (1995). "Automatic control issues in the development of an artificial pancreas," Proc. ACC, pp. 771-775.

[28] Kienitz, K. and Yoneyama, T. (1993). "Robust controller for insulin pumps based on H-infinity theory," IEEE Transactions on Biomedical Engineering, vol 40(11), pp. 1133-1137. 